\title{
Evolución química del agua subterránea a través del acuífero del Valle de Mexicali
}

\section{Chemical evolution of groundwater through the Mexicali Valley aquifer}

\author{
GÓMEZ-PUENTES, Francisco Javier ${ }^{1} \dagger^{*}$, REYES-LÓPEZ, Jaime Alonso ${ }^{2}$ y AMADO-MORENO, \\ María Guadalupe ${ }^{1}$
}

${ }^{1}$ Tecnológico Nacional de México/I.T. Mexicali, Av. Tecnológico S/N Col. Elías Calles, Mexicali, B.C., C.P. 21376

${ }^{2}$ Universidad Autónoma de Baja California / Instituto de Ingeniería, Blvd. Benito Juárez y Calle de la Normal s/n, Col. Insurgentes, Mexicali, B.C, CP 21280

ID 1 ${ }^{\text {er }}$ Autor: Francisco Javier, Gómez-Puentes / ORC ID: 0000-0002-8778-8173, CVU CONACYT ID: 228457

ID $1^{\text {er }}$ Coautor: Jaime Alonso, Reyes-López / ORC ID: 0000-0001-7962-9191, CVU CONACYT ID: 12637

ID $2^{\text {do }}$ Coautor: María Guadalupe, Amado-Moreno / ORC ID: 0000-0001-6363-5888, CVU CONACYT ID: 63559

\section{Resumen}

Se evaluó la calidad del agua del acuífero del Valle de Mexicali afín de estimar los procesos geoquímicos involucrados con el incremento de su salinidad y la subsecuente mineralización del subsuelo. El área de estudio se ubica en el municipio de Mexicali, Baja California, México, en un tramo donde el corredor ripario del río Colorado converge con una zona agrícola. El agua de este río contiene menos de 1000 ppm de sólidos disueltos en promedio, pero a medida que el agua es canalizada y utilizada en el riego de cultivos, experimenta un proceso de enriquecimiento de solutos hasta alcanzar 2400 ppm en el acuífero superior. De acuerdo con los análisis químicos, la salinización que presenta el agua es provocada principalmente por los iones sodio, sulfato y cloruro mientras que la modelación geoquímica realizada con el programa PHREEQC indica que los procesos relacionados con la precipitación de calcita, disolución de dolomita y yeso, y evaporación de agua, son los principales responsables de la evolución química del agua en la zona.

PHREEQC, Modelación geoquímica, Proceso de mineralización

\begin{abstract}
The water quality of the Mexicali Valley aquifer was evaluated in order to estimate the geochemical processes involved with the increase of its salinity and the subsequent subsoil mineralization. The study area is located in the municipality of Mexicali, Baja California, Mexico in a section where the riparian corridor of the Colorado River converges with an agricultural area. The water of this river contains less than $1000 \mathrm{ppm}$ of dissolved solids on average, but as the water is channeled and used in the crop's irrigation, it undergoes a solute enrichment process until $2400 \mathrm{ppm}$ in the upper aquifer. According to chemical analyzes the water salinization is mainly caused by sodium, sulfate and chlorine ions, while the geochemical modeling carried out with the PHREEQC program indicates that the processes related to calcite precipitation, dolomite and gypsum dissolution and water evaporation are primarily responsible for the water chemical evolution in the area.
\end{abstract}

PHREEQC, Geochemical modeling, Mineralization process

Citación: GÓMEZ-PUENTES, Francisco Javier, REYES-LÓPEZ, Jaime Alonso y AMADO-MORENO, María Guadalupe. Evolución química del agua subterránea a través del acuífero del Valle de Mexicali. Revista de Operaciones Tecnológicas. 2019. 3-9: 30-36

\footnotetext{
* Correspondencia del Autor (Correo electrónico: javiergomezp@itmexicali.edu.mx)

$\uparrow$ Investigador contribuyendo como primer autor.
} 


\section{Introducción}

En el Valle de Mexicali, las principales corrientes son: el Río Colorado, el Río Hardy y el Río Nuevo. El caudal del Río Colorado se ha visto controlado para la utilización del agua en actividades agrícolas, industriales y urbanas en el mismo delta y aguas arriba (Glenn et al., 1998). El río Colorado se origina en territorio estadounidense y desemboca en el golfo de California, cruzando aproximadamente $90 \mathrm{~km}$ en territorio mexicano. Su escurrimiento anual es de $1850 \mathrm{Mm}^{3}$, volumen que corresponde a la cuota asignada a nuestro país (RamírezHernández, 1997). Sus aguas se almacenan en la Presa Morelos, desde donde se distribuyen a través de un complejo sistema de canales.

En el valle de Mexicali coexisten dos acuíferos superpuestos y separados entre sí, de forma irregular, por una capa de sedimentos de baja permeabilidad. El nivel profundo es conocido como "yacimiento geotérmico de Cerro Prieto". El nivel más somero corresponde al acuífero superior, el cual tiene su nivel freático aproximadamente de 2 a 6 metros bajo la superficie del suelo. Este acuífero es de tipo libre y es considerado el de mayor capacidad en Baja California. El volumen de extracción es aproximadamente de $750 \mathrm{Mm}^{3} /$ año de agua, lo que representa una sobreexplotación de $50 \mathrm{Mm}^{3}$ en relacion con el volumen de recarga estimado (Ramírez-Hernández, 1997). La dirección de flujo regional del acuífero somero es de noreste a suroeste, con un gradiente hidráulico estimado en $0.042 \mathrm{~m} / \mathrm{km}$. Su agua se destina al uso agrícola y en menor escala al uso domesticourbano de la ciudad de Mexicali.

Por las características geológicas de ambiente deltaico, la principal explotación de los suelos del valle de Mexicali ha sido la agricultura, de modo que el riego de las tierras de cultivo se ha venido llevando a cabo mediante el aprovechamiento de las únicas fuentes de agua disponibles, las aguas del Río Colorado y las del acuífero de la región. Con éstas aguas se abastecen, a través de una amplia red de canales, 207,935 hectáreas de tierras agrícolas del Distrito de Riego 014. A partir de 1956 se empezó a hacer uso de fertilizantes en el valle de Mexicali. Para 1990, toda la superficie agrícola estaba fertilizada y en el periodo de 1982-1985 tan solo en el cultivo algodonero se aplicó un volumen total de 1,607,287 litros de insecticida (Ramírez-Hernández, 2006).
La calidad del agua del acuífero del Valle de Mexicali se ha degradado paulatinamente desde el inicio de su explotación. Al cambiar el régimen de recarga del acuífero, el cual el Río Colorado deja de ser el principal aportador y la infiltración vertical descendente se convierte en el proceso de recarga más importante. El agua infiltrada de parcelas y drenajes agrícolas sufre un proceso muy intenso de evaporación y, por tanto, de mineralización. Los volúmenes actuales de extracción de agua hacen necesario que el agua de infiltración migre rápidamente hacia capas más profundas y sea bombeada de nuevo a la superficie para su uso en la agricultura. Entre los años 1999 y 2002, se reportaron valores promedios, para el agua del acuífero, de $1307 \mathrm{mg} / \mathrm{L}$ de sólidos disueltos (Ramírez-Hernández, 2006).

Área de estudio. El sitio de estudio se localiza en el Valle de Mexicali, B.C. en el noroeste de México, esta enclavado dentro del corredor ripario del Río Colorado, a una distancia aproximada de un kilometro de su cauce. Las coordenadas geográficas correspondientes son: $32^{\circ} 12^{\prime}$ Latitud Norte y $115^{\circ}$ 09' Longitud Oeste (Figura 1). El sitio colinda al norte con varias parcelas de cultivo de riego por inundación siendo el asentamiento humano más cercano el poblado Oviedo Mota. Los cuerpos de agua superficiales más importantes en la zona de estudio son: (1) el río Colorado, ubicado en el extremo sur, (2) un canal para riego agrícola localizado en el extremo norte, y (3) el dren de desagüe agrícola.

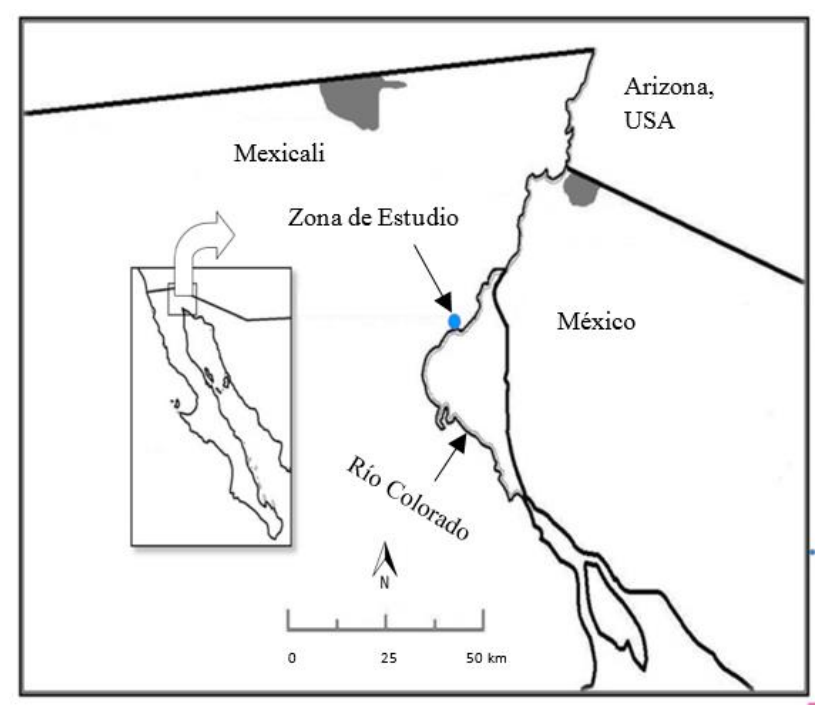

Figura 1 Localización del área de estudio 
La interacción agua superficial-agua subterránea en la zona de estudio se hace evidente por correspondencia entre la variación del nivel estático del acuífero con el ciclo agrícola. Durante los periodos de riego algunas porciones del río Colorado actúan como dren colector del agua subterránea, así como del agua en exceso transportada por el sistema de drenes agrícola (Pérez-González, 2008). Debido a la escasa precipitación en la región, el agua aplicada a las zonas de cultivo constituye la principal fuente de recarga al acuífero, convirtiéndose también en el principal componente del agua superficial de la zona.

\section{Metodología desarrollada}

Muestreo de agua. Se tomaron muestras de agua del Río Colorado, así como de 06 pozos de monitoreo de agua subterranea instalados en la proximidades de una parcela de cultivo ubicada en el Distrito de Riego 14 del Valle de Mexicali. Se realizaron 04 campañas bimestrales de muestreo durante el año 2017 (Figura 2).

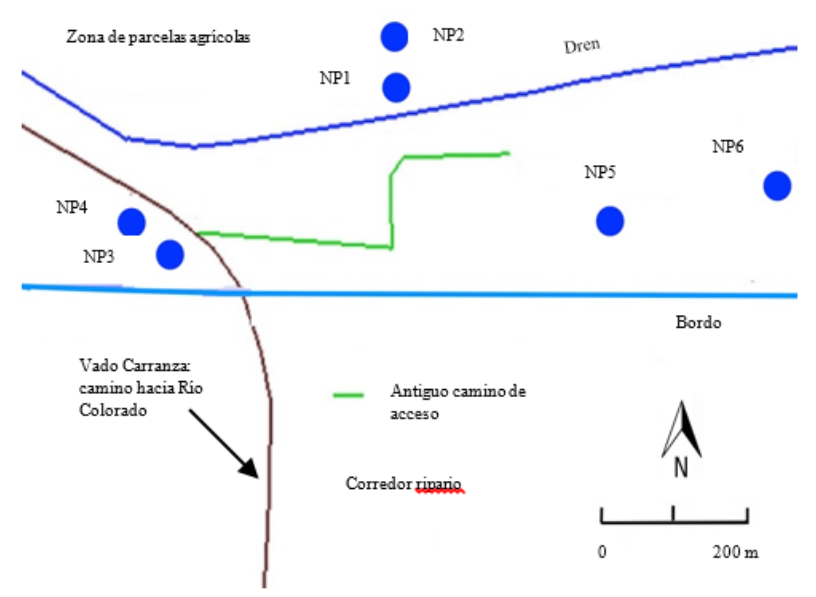

Figura 2 Ubicación espacial de los pozos de monitoreo de agua subterránea

Cada uno de los muestreos se realizó siguiendo las recomendaciones establecidas en la Norma Oficial Mexicana (Diario Oficial de la Federación, 1994). Las muestras de agua fueron colectadas mediante un sistema de vacío tipo manual (Fetter, Boving, \& Kreamer, 2017). Se realizó la medición del nivel freático del agua subterránea en cada uno de los pozos procediéndose posteriormente a su extracción, esta agua no formó parte de la muestra final, sino que se desecho y se permitió la recuperación del agua a su nivel original procediéndose al muestreo definitivo. Para cada estación, se tomaron muestras por duplicado en botellas de polietileno de $1 \mathrm{~L}$ de capacidad para cationes y aniones respectivamente.
Las muestras destinadas al análisis de cationes fueron acidificadas a un $\mathrm{pH}$ menor a dos unidades según las recomendaciones de la literatura (Appelo \& Postma, 2005). En la tabla 1 se indica la ubicación, en coordenadas UTM, de cada una de las estaciones de muestreo de agua subterránea.

\begin{tabular}{|l|c|c|}
\hline $\begin{array}{c}\text { Estaciones de } \\
\text { muestreo }\end{array}$ & \multicolumn{2}{c|}{ Coordenadas UTM } \\
\hline NP1 & 673715 & Y \\
\hline NP2 & 673715 & 3564638 \\
\hline NP3 & 673392 & 3564474 \\
\hline NP4 & 673351 & 3564492 \\
\hline NP5 & 674029 & 3564510 \\
\hline NP6 & 674266 & 3564535 \\
\hline
\end{tabular}

Tabla 1 Ubicación de los pozos de monitoreo de agua subterranea

Análisis de agua. En la tabla 2 se resumen las diferentes técnicas empleadas en el análisis de las muestras de agua. Las mediciones en campo se llevaron a cabo mediante instrumentos portátiles previamente calibrados e incluyeron los siguientes parámetros: $\mathrm{pH}$, conductividad eléctrica, solidos disueltos totales y oxígeno disuelto.

Los análisis de los aniones fueron realizados de acuerdo con los procedimientos recomendados por la APHA (American Public Health Association, 1965) así como por la norma mexicana correspondiente.

La cuantificación de cationes se realizó mediante la técnica de espectrometría de absorción atómica de flama de acuerdo con lo establecido por la norma mexicana NMX-AA051-SCFI-2001 (Secretaría de Economía, 2001).

El coeficiente de correlación de las distintas curvas de calibrado para cada uno de los cationes analizados estuvo por encima del valor 0.995 y en lo que respecta a la exactitud metodológica de los análisis, los porcentajes de recuperación estuvieron en el rango comprendido entre 95 y 105. 


\begin{tabular}{|c|c|c|}
\hline Parámetro & Método & Observaciones \\
\hline $\begin{array}{l}\text { Conductividad } \\
\text { eléctrica y } \\
\text { solidos disueltos }\end{array}$ & $\begin{array}{l}\text { Medidor de } \\
\text { conductividad, Marca } \\
\text { Oakton 35608 Series }\end{array}$ & $\begin{array}{l}\text { Medición } \\
\text { campo }\end{array}$ \\
\hline $\begin{array}{ll}\mathrm{pH} & \mathrm{y} \\
\text { temperatura }\end{array}$ & $\begin{array}{ll}\text { Medidor } & \text { de } \mathrm{pH} / \mathrm{ORP} \\
\text { Marca Hanna HI } \\
98140\end{array}$ & $\begin{array}{l}\text { Medición } \\
\text { campo }\end{array}$ \\
\hline $\begin{array}{l}\text { Alcalinidad, } \\
\mathrm{CO}_{3}^{-2}{\text { y } \mathrm{HCO}_{3}}^{-}\end{array}$ & Titulación de Gran & $\begin{array}{l}\text { Método } \\
\text { punto } \\
\text { inflexión }\end{array}$ \\
\hline Nitratos & $\begin{array}{l}\text { Método } \\
\text { Reducción de cadmio } \\
\text { (ESEPA 1980) }\end{array}$ & $\begin{array}{l}\text { Método } \\
\text { colorimétrico } \\
\text { Hach DR5000 } \\
\end{array}$ \\
\hline Cloruros & $\begin{array}{l}\text { Norma Mexicana } \\
\text { NMX-AA-073-SCFI- } \\
1981 \\
\end{array}$ & Método de Mohr \\
\hline Sulfatos & $\begin{array}{l}\text { Norma Mexicana } \\
\text { NMX-AA-074- SCFI- } \\
1981\end{array}$ & $\begin{array}{l}\text { Método } \\
\text { turbidimétrico }\end{array}$ \\
\hline $\begin{array}{l}\text { Cationes (Na, K, } \\
\text { Ca y Mg) }\end{array}$ & $\begin{array}{l}\text { Espectrometría de } \\
\text { Absorción Atómica }\end{array}$ & $\begin{array}{l}\text { Equipo GBC } \\
\text { modelo Avanta }\end{array}$ \\
\hline
\end{tabular}

Tabla 2 Métodos utilizados para el análisis químico del agua

Predicción de mineralización. Los datos obtenidos a partir de los análisis efectuados a las muestras de agua subterránea se emplearon para llevar a cabo una modelación geoquímica inversa mediante el programa (Parkhurst \& Appelo, 1999). Este tipo de modelación tiene por objetivo evidenciar los cambios químicos que experimenta un agua natural a través de una trayectoria de flujo. Se asume que un tipo de agua (solución origen) reacciona con minerales y gases para producir la composición química de un segundo tipo de agua (solución final).

La secuencia del proceso de modelación fue como sigue: 1) Los parámetros físicos y químicos de las dos soluciones seleccionadas (Río Colorado y pozo NP2) se introdujeron al programa, 2) Se procedió a obtener la especiación química de ambas soluciones con la finalidad de obtener los índices de saturación de cada una de las fases minerales asociadas, 3) La concentración del ion cloruro se igualo simulando un proceso de evaporación considerando el carácter conservativo de este ion, 4)

Se especificaron las incertidumbre asociada a los cálculos para cada solución, 5) Se seleccionaron las fases minerales para la modelación en base a la especiación química realizada y la paragénesis de minerales según la geología de la zona de estudio y 6) mediante la ejecución del programa se obtuvieron varios modelos, seleccionando el mejor de acuerdo a los criterios establecidos como balance de masa, incertidumbres y porcentaje de error.

\section{Resultados}

En la tabla 3 se presentan los valores promedio y desviación estándar de parámetros fisicoquímicos analizados para cada uno de los pozos de monitoreo de agua subterránea, así como del Río Colorado. Los valores promedio de la tabla señalada, corresponden a las diferentes campañas de muestreo

Parámetros fisicoquímicos. En general, los valores de $\mathrm{pH}$ tanto del agua del Río Colorado como del agua subterránea revelan aguas ligeramente alcalinas que en general caen dentro el rango promedio medio en aguas naturales continentales. Por otro lado, el contenido de sólidos disueltos en el agua subterránea, en general, superó en relación 3 a 1 al encontrado en el agua del Río Colorado en proporción ligeramente superior a 3:1 respecto a los pozos con el mayor contenido de solidos disueltos (NP1 y NP2), evidenciando el enriquecimiento en solutos debido al proceso natural de infiltración, así como a la actividad agrícola en la zona.

Mientras que el contenido de sólidos disueltos en el agua del Río Colorado fue de 760 $\pm 80 \mathrm{ppm}$, los pozos de monitoreo de agua subterránea identificados como NP1 y NP2 presentaron el mayor contenido. Estos pozos son los mas cercanos a las parcelas de cultivo de la zona. En el caso del oxígeno disuelto (OD) las concentraciones obtenidas estuvieron en los rangos generalmente medidos según el tipo de agua respectivo. Para el Río Colorado el valor promedio fue de $7.8 \mathrm{mg} / \mathrm{L}$ que es típico para un agua superficial en tanto que para el agua subterránea estuvo entre 1.5 a $2.8 \mathrm{mg} / \mathrm{L}$.

Aunque no queda evidenciado en la tabla 3, el OD sigue el patrón de comportamiento esperado de encontrarse en mayor concentración durante los meses de invierno. La marcada diferencia en el contenido de OD entre los dos tipos de agua puede explicarse dadas las condiciones reductoras prevalecientes en el acuífero. Finalmente, los valores correspondientes al potencial redox (ORP) confirman la diferencia entre las condiciones reducidas del acuífero a y las oxidadas de la superficie a pesar del nivel freático somero en la zona cuya profundidad máxima medida se encontró alrededor de los $3.5 \mathrm{~m}$ con relación a la superficie. 


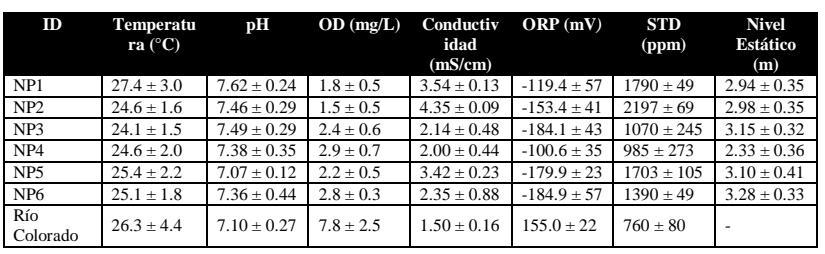

Tabla 3 Valores promedio y desviacion estandar de los parametros fisicoquimicos medidos en agua

Iones Mayoritarios. En el caso del Río Colorado, los resultados obtenidos revelan que el sodio es el catión más abundante con una concentración promedio de $252 \mathrm{mg} / \mathrm{L}$, seguido del magnesio $(64 \mathrm{mg} / \mathrm{L})$, el calcio $(75 \mathrm{mg} / \mathrm{L})$ y por último el potasio $(4.7 \mathrm{mg} / \mathrm{L})$. Por otro lado, el anión más abundante en esta agua es el sulfato $(370 \mathrm{mg} / \mathrm{L})$, seguido por los cloruros que presentan una concentración media de 340 $\mathrm{mg} / \mathrm{L}$. En el caso del agua subterránea, para el pozo con el mayor contenido de solidos disueltos (NP2) el sodio resultó ser el catión más abundante con un contenido que alcanzo 514 $\mathrm{mg} / \mathrm{L}$, seguido del magnesio $(82 \mathrm{mg} / \mathrm{L})$, el calcio $(95 \mathrm{mg} / \mathrm{L})$ y el potasio $(6.6 \mathrm{mg} / \mathrm{l})$.

Por lo que los resultados obtenidos evidencian el enriquecimiento progresivo de solutos a medida que el agua superficial del Río Colorado se infiltra para abastecer al acuífero del valle de Mexicali. Además, a dicho enriquecimiento contribuyen la sobreexplotación y reúso del agua del acuífero en actividades de riego agrícola, así como el proceso de evaporación que experimenta el agua durante la epoca de verano. Un patrón similar se observa con los resultados obtenidos de los aniones en los que el más dominante resulto ser el sulfato, seguido del cloro.

Estas características revelan aguas de tipo sulfatadas cloruradas sódicas tanto para el Río Colorado como para el agua del acuífero del Valle de Mexicali. En la figura 3, las relaciones iónicas de $\mathrm{Na}$ y $\mathrm{Cl}$ ponen en evidencia el enriquecimiento de solutos y mas específicamente el proceso de salinización a medida que el agua subterránea evoluciona químicamente, siguiendo su dirección de flujo, a partir del agua del Río Colorado (RC) a partir de la cual se origina y que posee el menor contenido de solidos disueltos hasta los pozos (NP1 y NP2) mas concentrados y que están en las inmediaciones de las parcelas de cultivo de la zona.

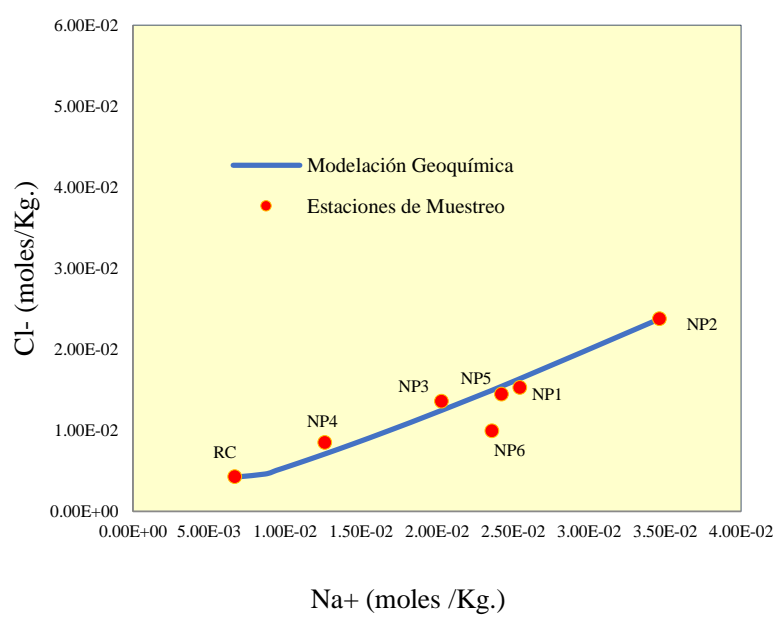

Figura 3 Relaciones ionicas entre los iones sodio y cloruro correspondientes al muestreo de abril de 2017

Predicción de mineralización. En la figura 4 se presenta los resultados obtenidos de la especiación química para las muestras de agua del Río Colorado y del pozo NP2 que resultó con la mayor concentración de solidos disueltos. Se presentan los índices de saturación (IS) para ambos tipos de agua. Se distingue claramente que los IS mas elevados corresponden al pozo NP2, alcanzando valores positivos para los minerales aragonito $\left(\mathrm{CaCO}_{3}\right)$, calcita $\left(\mathrm{CaCO}_{3}\right)$ y dolomita $\left(\mathrm{CaMgCO}_{3}\right)_{2}$.

Estos valores predicen la tendencia a precipitar de estas fases minerales debido a la cantidad excesiva de los iones que les dan origen. Este incremento en los iones mayoritarios puede deberse tanto a las interacciones entre agua-roca conforme el agua del Río Colorado se infiltra y se desplaza en el subsuelo como también a los procesos de evaporación de agua debido a las características semiáridas del clima asociado a la zona de estudio.

Por otro lado, no menos importante es el incremento en el índice de saturación que experimenta el mineral halita, el cual a pesar de que conserva su valor negativo en agua subterránea evidencia su enriquecimiento de los iones $\mathrm{Na}$ y $\mathrm{Cl}$. El valor negativo persistente de estos iones en agua subterránea son indicativos su elevada solubilidad. 


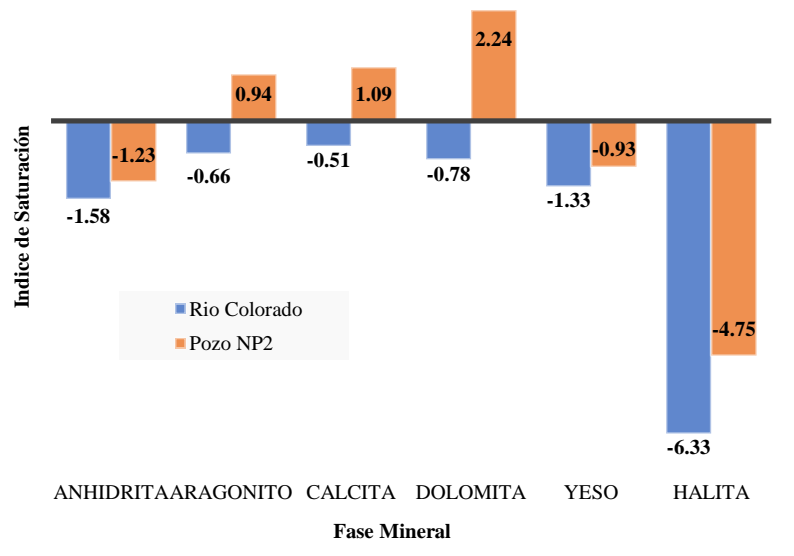

Figura 4 Índices de saturación de las fases minerales para el agua del Río Colorado y el pozo de monitoreo NP2

Finalmente, la modelación inversa realizada permitió reproducir la evolución química del agua subterránea a partir de un agua con las características del Río Colorado. La tabla 4 sintetiza los resultados mas relevantes de esta modelación: la solución origen consiste en el agua superficial del Rio Colorado que da origen a la solución final consistente en agua subterránea con las características químicas observadas en el pozo NP2. Aunque los resultados corresponden a la campaña de muestreo de octubre de 2017, para el resto de las campañas se obtuvieron esencialmente los mismos resultados.

Los valores en porcentaje para las incertidumbres indican que el programa PHREEQC permite hasta una variación de $\pm 5 \%$ y de $\pm 7.3 \%$ en las cantidades que son utilizadas en los cálculos iterativos de cada uno de los parámetros de las respectivas soluciones. En cada modelación se emplearon los valores de incertidumbre mas bajos posibles. Con base en la especiación química y a la paragénesis de los minerales en la zona de estudio se seleccionaron las fases minerales.

A partir de la modelación se obtuvieron los procesos y las cantidades presentadas en la tabla 4. En la parte final de la de esta, se presenta la comparación entre las dos soluciones obteniéndose porcentajes de error menores al 5\% entre la muestra real NP2 y la solución obtenida a partir de la modelación. El modelo obtenido predice los siguientes procesos geoquímicos como los más importantes: disolución de los minerales halita, dolomita, y yeso, precipitación del mineral calcita, y la evaporación de agua.

\begin{tabular}{|c|c|c|c|}
\hline Denominación & Tipo de Agua & \multicolumn{2}{|l|}{ Incertidumbre } \\
\hline $\begin{array}{l}\text { Solución origen: } \\
\text { Río Colorado }\end{array}$ & Superficial & & $5.0 \%$ \\
\hline $\begin{array}{l}\text { Solución final: } \\
\text { Pozo NP2 }\end{array}$ & Subterránea & & $7.3 \%$ \\
\hline \multicolumn{4}{|c|}{ 2. Resultados de la Modelización } \\
\hline Fase Mineral & Proceso & \multicolumn{2}{|c|}{ Cantidad (mol/kg H2O) } \\
\hline Halita & Disolución & & 0.25 \\
\hline $\mathrm{H}_{2} \mathrm{O}(\mathrm{g})$ & Evaporación & & 1.53 \\
\hline Calcita & Disolución & & $5.4 \times 10^{-2}$ \\
\hline Dolomita & Precipitación & & $2.2 \times 10^{-2}$ \\
\hline Yeso & Disolución & & $3.2 \times 10^{-2}$ \\
\hline \multicolumn{4}{|c|}{ 3. Comparación de soluciones } \\
\hline Parámetro & $\begin{array}{l}\text { NP2 (Solución } \\
\text { Real) }\end{array}$ & $\begin{array}{c}\text { NP2 } \\
\text { (Solución } \\
\text { Modelada) }\end{array}$ & \% Error \\
\hline $\mathrm{pH}$ & 7.5 & 7.5 & 0.03 \\
\hline $\mathrm{HCO}_{3}{ }^{-}$ & $2.6 \times 10^{-2}$ & $2.4 \times 10^{-2}$ & 4.6 \\
\hline $\mathrm{Ca}$ & $2.5 \times 10^{-3}$ & $2.5 \times 10^{-3}$ & 0.0 \\
\hline $\mathrm{Cl}$ & $2.6 \times 10^{-1}$ & $2.4 \times 10^{-1}$ & 3.7 \\
\hline $\mathrm{K}$ & $2.3 \times 10^{-3}$ & $2.3 \times 10^{-3}$ & 0.0 \\
\hline $\mathrm{Na}$ & $2.8 \times 10^{-1}$ & $3.0 \times 10^{-1}$ & 3.5 \\
\hline $\mathrm{Mg}$ & $1.4 \times 10^{-2}$ & $1.5 \times 10^{-2}$ & 3.5 \\
\hline $\mathrm{SO}_{4}^{-}$ & $4.0 \times 10^{-2}$ & $3.8 \times 10^{-2}$ & 3.9 \\
\hline
\end{tabular}

Tabla 3 Resultados de la modelación geoquímica inversa (Río Colorado - agua del acuifero superior del Valle de Mexicali)

La calcita es un mineral común principalmente en Europa y Norteamérica (Appelo \& Postma, 2005). Análisis químicos de suelo realizados en la zona de estudio revelaron concentraciones de carbonatos de calcio y magnesio de alrededor de $8 \%$. Asimismo, análisis cualitativos en suelo revelaron la ocurrencia de yeso en la zona de estudio. En el caso del mineral halita su no ocurrencia en la zona permite inferir que su origen es antropogénico.

\section{Agradecimiento}

Al Tecnologico Nacional de México (TecNM) por el financiamiento otorgado para la realización de la presente investigación.

\section{Conclusiones}

El agua del Río Colorado contiene un promedio de 760 ppm de sólidos disueltos. A medida que esta agua es canalizada y utilizada preferentemente en el riego de cultivos experimenta un proceso de enriquecimiento en solutos hasta alcanzar concentraciones cercanas a los 2400 ppm en el acuífero del Valle de Mexicali. La química de estas aguas esta dominada por los iones Sodio, Sulfato y Cloruro. 
Los procesos geoquímicos más importantes que explican la evolución química del Río Colorado, de acuerdo con la modelización realizada con el programa PHREEQC, consisten en la precipitación de calcita, disolución de dolomita y yeso, evaporación de agua, así como en la salinización provocada por el enriquecimiento de los iones $\mathrm{Na}, \mathrm{Cl}$ y $\mathrm{SO}_{4}^{-2}$. La no ocurrencia natural del mineral halita en la zona de estudio permite establecer que las altas concentraciones de sodio y cloro en agua subterránea tienen un origen antropogénico.

\section{Referencias}

American Public Health Association. (1965). Standard methods for the examination of water and wastewater: Including bottom sediments and sludges. New York.

Appelo, C. A. J., \& Postma, D. (2005). Geochemistry, Groundwater and Pollution (2a Ed.). the Netherlands: A. A. Balkema Publishers.

Diario Oficial de la Federación. (1994). Norma Oficial Mexicana NOM-014-SSA1-1993, Procedimientos sanitarios para el muestreo de agua para uso y consumo humano en sistemas de abastecimiento de agua publicos y privados. Recuperado el 10 de septiembre de 2019, de http://www.dof.gob.mx/nota_to_imagen_fs.php ?codnota $=4728763 \&$ fecha $=12 / 08 / 1994 \&$ cod_di ario $=203795$

Fetter, C. W., Boving, T., \& Kreamer, D. (2017). Contaminant Hydrogeology: Third Edition (3a Ed.). USA: Waveland Press.

Glenn, E., Tanner, R., Mendez, S., Kehret, T., Moore, D., Garcia, J., \& Valdes, C. (1998). Growth rates, salt tolerance and water use characteristics of native and invasive riparian plants from the delta of the Colorado River, Mexico. Journal of Arid Environments, 40(3), 281-294.

https://doi.org/10.1006/jare.1998.0443

Parkhurst, L., \& Appelo, C. A. J. (1999). User's Guide to PHREEQC (Version 2)-A Computer Program for Speciation, Batch-Reaction, OneDimensional Transport, and Inverse Geochemical Calculations. Recuperado el 9 de septiembre de 2019, de https://wwwbrr.cr.usgs.gov/projects/GWC_cou pled/phreeqc/html/final.html

ISSN 2523-6806

ECORFAN® Todos los derechos reservados
Pérez-González, D. (2008). Interacción Hidrodinámica del Río Colorado y el Acuífero del Valle de Mexicali en el Tramo FFCC. - Vado Col. Carranza, México. (Tesis de Maestría). Universidad Autonoma de Baja California, Mexicali, B.C.

Ramírez-Hernández, J. (1997). Estudio de las relaciones geohidrológicas del acuífero superior del valle de Mexicali con aguas geotermicas superficiales. Universidad de Alcalá, Madrid.

Ramírez-Hernández, J. (2006). Una visión de la problemática ambiental de Mexicali y su valle: Elementos para su gestión. UABC.

Secretaría de Economía. (2001). NMX-AA-051SCFI-2001 Análisis de agua-Determinación de metales por absorción atómica en aguas naturales, potables, residuales y residuales tratadas-Método de prueba. Recuperado el 10 de septiembre de 2019, de https://agua.org.mx/biblioteca/nmx-aa-051-scfi2001-analisis-de-agua-determinacion-demetales-por-absorcion-atomica-en-aguasnaturales-potables-residuales-y-residualestratadas-metodo-de-prueba/ 